\title{
La aceptabilidad de un protocolo de tratamiento transdiagnóstico autoaplicado a través de Internet: datos preliminares
}

\author{
Alberto GonZÁlez Robles \\ vrobles@uji.es \\ AMANDA DíAz GARCÍA \\ adiazgs8@gmail.com \\ MARÍA SOl FITTIPALDI MÁRQUEZ \\ al283815@uji.es \\ Cristina Botella Arbona \\ botella@uji.es
}

\section{Resumen}

Introducción: En los últimos años ha habido un interés creciente en abordar el tratamiento de los trastornos emocionales (TE) desde una perspectiva transdiagnóstica. Los protocolos transdiagnósticos enfatizan los procesos esenciales subyacentes a los distintos TE, son adecuados para el tratamiento de distintos trastornos psicológicos y permiten un abordaje más adecuado de la comorbilidad entre este tipo de trastornos. Por otra parte, el uso de las tecnologías de la información y la comunicación (TIC) puede ayudar a que los tratamientos psicológicos lleguen a un mayor número de personas, contribuyendo de esta manera a mejorar su eficiencia. La mayoría de trabajos sobre protocolos de tratamiento online se centran en el estudio de su eficacia, sin embargo es importante también estudiar la aceptabilidad de este tipo de intervenciones. Nuestro grupo de investigación ha desarrollado un protocolo de tratamiento transdiagnóstico para los TE, y lo ha adaptado para que pueda ser aplicado a través de Internet. En este trabajo se describe este protocolo y se presentan datos preliminares sobre su aceptabilidad. Metodología: 12 participantes (edad media $=28,58$, d. t. $=5,35)$ de una muestra clínica con un diagnóstico de TE que recibieron un protocolo de tratamiento transdiagnóstico combinado (terapia presencial + protocolo de tratamiento online). Se obtuvieron datos acerca de las expectativas y opinión de los participantes evaluadas con la escala de expectativas sobre el tratamiento y la escala de opinión sobre el tratamiento. Resultados: Las puntuaciones obtenidas fueron elevadas en ambas variables relacionadas con la aceptación (expectativas y opinión). Discusión: El estudio de la aceptabilidad por parte de los pacientes hacia estos programas es importante, ya que puede influir en la decisión acerca de iniciar y/o seguir este tipo de tratamientos. Contar con esta información puede ayudarnos 
en el desarrollo y mejora de los tratamientos psicológicos autoaplicados a través de Internet.

Palabras clave: trastornos emocionales, transdiagnóstico, tecnologías de la información y la comunicación, Internet, aceptabilidad.

\begin{abstract}
Introduction: In recent years there has been great interest in addressing the treatment of ED from a transdiagnostic perspective. Transdiagnostic treatment protocols emphasize the essential underlying processes that are common to ED and are conceived of for the treatment of several mental disorders. Also, comorbidity among these disorders can be more adequately addressed from this perspective. In addition, Information and Communication Technologies (ICTS) can facilitate access by people for whom traditional therapy is not available thereby contributing to an improvement in its cost-effectiveness. Most of the articles about online treatment protocols are efficacy studies, nevertheless it is also important to analyze the acceptability of these types of interventions. Our research group has developed a transdiagnostic treatment protocol for the treatment of ED that has been adapted to be applied online over the Internet. The aim of this article is to describe the treatment protocol and to present data about its acceptability. Methodology: 12 participants (mean age $=28.58$, d. t. $=5.35$ ) from a clinical sample with a diagnosis of ED which received a blended transdiagnostic protocol treatment (face-toface therapy plus online treatment protocol). Scores of expectancies and opinion of treatment were assessed with the Expectancies Treatment Scale and the Opinion Treatment Scale. Results: High scores in both variables related to acceptability (expectations and opinion of treatment) were obtained. Discussion: The study of the acceptability towards this kind of programs is important, since it may influence the participant's decision to initiate and/or follow this kind of treatments. Counting on this information can help us in the development and improvement of Internet-based treatment protocols.
\end{abstract}

Keywords: emotional disorders, transdiagnostic, information and communication technologies, Internet, acceptance.

\title{
Introducción
}

Los TE se encuentran entre los trastornos mentales más frecuentes, con una prevalencia vital del $29 \%$ y tasas de comorbilidad de entre el 40 y el $80 \%$ (Kessler y cols., 2005; Wittchen y cols., 2011). Estos datos apuntan claramente a la necesidad de disponer de tratamientos eficaces y eficientes que permitan hacer frente a este importante problema de salud.

En los últimos 20 años, se han desarrollado y validado un importante número de tratamientos cognitivo-conductuales basados en la evidencia (Hollon, y Ponniah, 2010; Nathan, y Gorman, 2007; Norton, y Price, 2007; Olatunji, Cisler, y Deacon, 2010). Sin embargo, se trata de protocolos de tratamiento específicos, cada uno de ellos orientado al tratamiento de un determinado trastorno psicológico. 
Recientemente, se observa un interés creciente en abordar el tratamiento de los TE desde una perspectiva transdiagnóstica. Esta perspectiva enfatiza los procesos esenciales subyacentes a los distintos TE. Barlow, Allen, y Choate (2004) propusieron el Modelo de la triple vulnerabilidad para explicar la existencia de una vulnerabilidad biológica y psicológica, que es común a los distintos TE, y que ayudaría a explicar la comorbilidad entre este tipo de trastornos mentales. Además, este modelo teórico ha servido de base a este mismo grupo para desarrollar el Protocolo Unificado (PU) (Barlow y cols., 2004), un protocolo de tratamiento transdiagnóstico que enfatiza el papel de la regulación emocional en la comprensión y el tratamiento de los TE. Este protocolo, a diferencia de los protocolos específicos, más centrados en abordar los síntomas particulares de cada trastorno mental, permite abordar el tratamiento de los TE poniendo el énfasis en las vulnerabilidades compartidas por estos trastornos. Existen resultados que han mostrado la eficacia de este protocolo en formato de terapia cara a cara (Bullis, Fortune, Farchione, y Barlow, 2014; Ellard, Fairholme, Boisseau, Farchione, y Barlow, 2010; Farchione y cols., 2012). Además, estudios de metaanálisis muestran que los protocolos de tratamiento transdiagnósticos producen resultados similares a los protocolos de tratamiento específicos basados en la evidencia (McEvoy, Nathan, y Norton, 2009).

Las TIC, en concreto Internet, han demostrado ser una herramienta prometedora en el tratamiento de distintos problemas mentales y en la diseminación de los tratamientos basados en la evidencia (Andersson, y Cuijpers, 2009; Clark y cols., 2009). Además, distintos metaanálisis han mostrado una mayor eficacia de este tipo de intervenciones frente a distintos grupos control (Andersson, y Cuijpers, 2009; Andrews, Cuijpers, Craske, McEvoy, y Titov, 2010) y también que son, al menos, tan eficaces como los tratamientos tradicionales cara a cara (Cuijpers, van Straten, y Andersson, 2008; Richards, y Richardson, 2012). Ahora bien, la mayoría de los estudios sobre protocolos de tratamientos computerizados (p. ej., tratamientos auto-aplicados a través de Internet) se centran en el estudio de su eficacia y existen pocos estudios centrados en variables relacionadas con la aceptabilidad (p. ej., expectativas y opinión) de este tipo de intervenciones. Sin embargo, el estudio de estas variables es importante, ya que están relacionadas con los resultados terapéuticos (Kaltenthaler y cols., 2008), y su estudio puede ayudar a mejorar la efectividad de estos programas (De Graaf, Huibers, Riper, Gerhards, y Arntz, 2009) y a predecir la respuesta a este tipo de intervenciones (Osgood-Hynes y cols., 1998).

Nuestro grupo de investigación ha desarrollado un protocolo de tratamiento transdiagnóstico basado en el PU de D. H. Barlow (Barlow y cols., 2004), y que incluye componentes de regulación emocional de la terapia dialéctico-comportamental de Marsha Linehan (Linehan, 2003), y lo ha adaptado para que pueda ser administrado a través de Internet. Los objetivos de este trabajo son: 1) describir el protocolo de tratamiento transdiagnóstico online; y 2) presentar datos preliminares sobre la aceptabilidad de este protocolo de tratamiento. En concreto, se presentan datos sobre expectativas y opinión como indicadores de la aceptabilidad.

\section{Método}

\section{Intervención}

La intervención consiste en un protocolo de tratamiento transdiagnóstico autoaplicado a través de Internet. El protocolo incluye los siguientes componentes terapéuticos (ver tabla 1): aumentar la conciencia emocional centrada en el momento presente y la aceptación de las emociones, facilitar y potenciar la flexibilidad cognitiva, identificar y modificar los patrones desadaptativos de evitación emocional y comportamental, y promover la exposición a las experiencias emocionales (incluyendo exposición interoceptiva y situacional). 
El protocolo permite abordar los siguientes TE: trastorno depresivo mayor (TDM), distimia (D), trastorno de pánico (TP), agorafobia (A), trastorno de ansiedad social (TAS), trastorno de ansiedad generalizada (TAG), trastorno obsesivo-compulsivo (TOC), trastorno de ansiedad no especificado (TANE) y trastorno del estado de ánimo no especificado (TEANE).

Cada módulo incluye distintos ejercicios y tareas con el objetivo de aprender y practicar estrategias de regulación emocional adaptativas, todo ello desde una perspectiva transdiagnóstica.

El protocolo incluye, además de los 12 módulos de tratamiento, un módulo inicial de bienvenida que proporciona al usuario información general sobre el protocolo y sus objetivos, así como recomendaciones para obtener el máximo beneficio del programa.

El programa también tiene las siguientes características: a) cada módulo incluye una serie de preguntas de opción múltiple que permite a los participantes evaluar su comprensión del módulo y les ayuda a decidir si necesitan revisar los contenidos; $b$ ) el programa permite a los participantes revisar los módulos tantas veces como necesiten; y $c$ ) la plataforma ofrece la posibilidad al usuario de monitorizar sus progresos mediante distintas gráficas (p. ej., niveles de ansiedad y depresión antes y después de recibir la intervención, y tras cada módulo de tratamiento).

Tabla 1

Módulos del protocolo de tratamiento

\footnotetext{
1. Los trastornos emocionales y la regulación emocional

2. Motivación para el cambio
}

3. Comprendiendo el papel de las emociones

4. Aceptando las experiencias emocionales

5. Practicando la aceptación

\section{Aprendiendo a flexibilizar}

7. Practicando la flexibilidad cognitiva

8. Evitación de las emociones

9. Conductas impulsadas/dirigidas por emociones
Informar acerca de la importancia de la regulación emocional en los trastornos emocionales.

Analizar las ventajas y desventajas del cambio, enfatizar la importancia de estar motivado y subrayar la importancia de establecer objetivos de vida significativos.

Informar acerca de la naturaleza y función de las emociones. Se explica el modelo de los tres componentes de la emoción.

Enseñar al paciente la aceptación de las experiencias emocionales y su importancia en el tratamiento.

Continuar aprendiendo la aceptación de las experiencias emocionales e incrementar la capacidad para darse cuenta de sus sensaciones, pensamientos y emociones.

Se centra en las trampas de pensamiento y la flexibilidad cognitiva, su importancia y la forma de potenciarla.

Enseña a identificar y modificar patrones de pensamiento desadaptativos para incrementar la flexibilidad cognitiva.

Se centra en cómo las estrategias de evitación emocional contribuyen al mantenimiento de los trastornos emocionales.

Familiarizarse con el concepto conductas impulsadas/dirigidas por emociones (CIDE), aprender a reconocerlas y reemplazarlas por la conducta opuesta a la emoción. 
10. Aceptando y afrontando las sensaciones físicas

11. Atreviéndonos: afrontando las emociones

12. Prevención de recaídas
Aprender a identificar las sensaciones físicas desencadenantes del malestar y a aumentar la tolerancia hacia ellas. Exposición interoceptiva.

Aprender a construir jerarquías de exposición para aumentar la tolerancia a las emociones y reducir las conductas de evitación.

Revisar las estrategias aprendidas a lo largo del programa, y enseñar al paciente a identificar y hacer frente a futuras situaciones de riesgo.

\section{Participantes}

Los participantes fueron 12 pacientes pertenecientes a una muestra comunitaria de entre 21 y 61 años (edad media $=28,58$; d. t. $=5,35)(71,43 \%$ mujeres). El diagnóstico principal de cada participante se obtuvo con la entrevista internacional neuropsiquiátrica MINI (Sheehan y cols., 1998). Dichos diagnósticos se distribuyeron de la siguiente manera: 5 participantes con TAG, 3 participantes con TDM, 1 participante con TAS, 1 participante con TP, 1 participante con A, y 1 participante con $\mathrm{D}$. Los participantes forman parte de un estudio controlado aleatorizado en el que se está estudiando la eficacia de un protocolo de tratamiento transdiagnóstico blended (terapia presencial más terapia online) y autoaplicado a través de Internet.

\section{Medidas}

Escala de expectativas sobre el tratamiento (EET) y Escala de opinión sobre el tratamiento (EOT) (Borkovec, y Nau, 1972). Son dos escalas de 5 ítems que proporcionan una medida subjetiva de las expectativas/opinión del paciente hacia el tratamiento que va a recibir. El contenido de estos 5 ítems, valorados en una escala de 0 a 10 y con una puntuación máxima de 50, evalúa los siguientes aspectos: lo lógico que les parece el tratamiento, en qué medida satisface al paciente, en qué medida recomendarían el tratamiento a una persona con el mismo problema, en qué medida creen que se podría utilizar para tratar otros problemas psicológicos, y su utilidad para el problema específico del paciente. La EET se aplica antes de comenzar el tratamiento, una vez se ha explicado al paciente en qué va a consistir el mismo (después del módulo de bienvenida y antes de iniciar el tratamiento). Su objetivo es medir la expectativa subjetiva del paciente acerca del tratamiento. La EOT se administra cuando el paciente ha completado el tratamiento y su objetivo es evaluar la satisfacción con el mismo.

\section{Resultados}

Los resultados sobre expectativas y opinión del tratamiento se muestran en la tabla 2 y la tabla 3, respectivamente. 
Tabla 2

Media y desviación típica para los ítems de la Escala de expectativas sobre el tratamiento

\begin{tabular}{ll}
\hline Ítem & Media (d. t.) \\
\hline 1. ¿En qué medida te parece lógico este tratamiento? & $8,29(1,40)$ \\
2. ¿En qué medida te satisface el tratamiento que vas a recibir? & $8,50(1,40)$ \\
3. ¿En qué medida le recomendarías este tratamiento a un amigo que tuviera & \\
$\quad$ tu mismo problema? & $8,79(1,25)$ \\
4. ¿En qué medida crees que este tratamiento podría ser útil para tratar otros & \\
$\quad$ problemas psicológicos? & $7,93(1,14)$ \\
5. ¿En qué medida crees que este tratamiento va a resultar útil en tu caso? & $8,07(1,64)$ \\
\hline
\end{tabular}

Tabla 3

Media y desviación típica para los ítems de la Escala de opinión sobre el tratamiento

\begin{tabular}{ll}
\hline Ítem & Media (d. t.) \\
\hline 1. ¿En qué medida te ha parecido lógico este tratamiento? & $8,77(1,09)$ \\
2. ¿En qué medida estás satisfecho con el tratamiento recibido? & $8,70(1,11)$ \\
3. ¿En qué medida le recomendarías este tratamiento a un amigo que tuviera & \\
$\quad$ tu mismo problema? & $9,31(0,09)$ \\
4. ¿En qué medida crees que este tratamiento podría ser útil para tratar otros & \\
$\quad$ problemas psicológicos? & $8,46(1,61)$ \\
5. ¿En qué medida crees que este tratamiento ha resultado útil en tu caso? & $8,85(1,07)$ \\
\hline
\end{tabular}

\section{Discusión y conclusiones}

Como puede observarse en los resultados, los participantes presentan puntuaciones elevadas en todos los ítems relacionados con expectativas sobre el tratamiento (puntuaciones entre 7,93 y 8,79 sobre 10): lógica del tratamiento, satisfacción con el tratamiento, recomendación del tratamiento a otras personas con problemas similares, utilidad del tratamiento para otros problemas psicológicos y utilidad del tratamiento para su problema específico.

Tras recibir el tratamiento, la satisfacción del programa no es solo elevada, sino que mejora respecto a las expectativas en todos los ítems (puntuaciones entre 8,46 y 9,31 sobre 10). Las puntuaciones obtenidas en cuanto a expectativas y opinión del protocolo de tratamiento sugieren que es bien aceptado por los pacientes y están en línea con las revisiones sistemáticas que indican que los tratamientos cognitivo-comportamentales computerizados cuentan con altos niveles de aceptabilidad, expectativas positivas y alta satisfacción con el tratamiento (Andrews y cols., 2010; Kaltenthaler y cols., 2008).

Las principales limitaciones de este trabajo son el reducido tamaño muestral empleado (de solo 12 participantes) y que se trata de un estudio piloto inicial con ausencia de grupo control. Por lo tanto, antes de sacar conclusiones más concluyentes y poder generalizar estos resultados a la población es necesario estudiar la aceptabilidad con muestras mayores que la utilizada en este estudio. 
Aunque los datos preliminares mostrados en este trabajo indican que el programa de tratamiento cuenta con buena aceptación por parte de los participantes, es necesario estudiar las variables relacionades con la aceptabilidad en estudios controlados aleatorizados. Este programa de tratamiento se está estudiando en este momento mediante un ensayo controlado aleatorizado en nuestro grupo de investigación, por lo que en el futuro dispondremos de datos que nos permitan sacar conclusiones más sólidas acerca de su aceptabilidad.

Por otro lado, aunque las revisiones sistemáticas muestran que los tratamientos cognitivo-conductuales computerizados son bien aceptados en general (p. ej. Andersson y cols., 2010), la terapia computerizada ( $\mathrm{y}$ en especial los tratamientos autoaplicados a través de Internet) supone un campo de estudio bastante nuevo, por lo que es importante continuar investigando las variables relacionadas con la aceptabilidad, ya que esta información puede ser de gran ayuda en el desarrollo y mejora de este tipo de programas de tratamiento. Por ejemplo, dado que las intervenciones online se realizan mediante TIC, puede ser importante diseñar estrategias de evaluación para estudiar variables que puedan incidir o perturbar la terapia, como la usabilidad del programa de tratamiento, el perfil de los usuarios en función de su experiencia previa con las TIC, o la aceptabilidad del propio sistema.

Por último, la realización de estudios con tratamientos blended puede proporcionar información muy valiosa acerca de la aceptabilidad de los protocolos de tratamiento auto-aplicados a través de Internet. Los tratamientos blended (combinados) implican la combinación de terapia presencial y terapia online (p. ej., Kooistra y cols., 2014). Recibir ambos formatos de terapia al mismo tiempo proporciona al investigador la oportunidad de evaluar distintos aspectos relacionados con la aceptabilidad que serían más difíciles de estudiar cuando se aplica únicamente uno de estos dos formatos de terapia ( $p$. ej. en qué medida cree el paciente que los contenidos abordados en la terapia presencial quedan adecuadamente reflejados en los módulos del protocolo online o en qué medida cree el paciente que únicamente la utilización del protocolo online podría ayudarle a resolver sus problemas prescindiendo de las sesiones presenciales con el terapeuta).

En conclusión, aunque la importancia del estudio de la eficacia de las intervenciones online está fuera de toda duda, resulta igualmente importante estudiar su aceptabilidad. La terapia online supone una aproximación nueva, con cambios considerables respecto a la terapia tradicional (la ausencia de un terapeuta o la propia autoaplicabilidad de la terapia son ejemplos de ello). Todo ello puede tener una importante repercusión en la forma en que es aceptada este tipo de terapia. Por todo ello, consideramos que la investigación en este sentido debe centrarse en responder preguntas como las siguientes ¿Cómo podemos mejorar los tratamientos psicológicos autoaplicados a través de Internet para aumentar su aceptabilidad entre la población? ¿Qué características (p. ej., de contenido y de usabilidad) debe reunir un protocolo de tratamiento online para resultar exitoso entre los pacientes que lo utilizan? ¿Es la presencia de un terapeuta un requisito fundamental para que una terapia psicológica pueda funcionar? ¿Qué actitudes y creencias tiene la población hacia esta forma de intervenciones online?

\section{Referencias bibliográficas}

American Psychological Association (2000). Diagnostic and statistical manual of mental disorders (4th ed. rev.). Washington, DC: American Psychological Association.

Andersson, G., y Cuijpers, P. (2009). Internet-based and other computerized psychological treatments for adult depression: A meta-analysis. Cognitive Behaviour Therapy, 38, 196205. 
Andrews, G., Cuijpers, P., Craske, M. G., McEvoy, P., y Titov, N. (2010). Computer therapy for the anxiety and depressive disorders is effective, acceptable and practical health care: a meta-analysis. PloS One, 5, e13196.

Barlow, D. H., Allen, L. B., y Choate, M. L. (2004). Toward a unified treatment for emotional disorders. Behavior Therapy, 35, 205-230.

Borkovec, T. D., y Nau, S. D. (1972). Credibility of analogue therapy rationales. Journal of Behavior Therapy and Experimental Psychiatry, 3, 257-260.

Bullis, J., Fortune, M., Farchione, T., y Barlow, D. H. (2014). A preliminary investigation of the long-term outcome of the Unified Protocol for Transdiagnostic Treatment of Emotional Disorders. Comprehensive Psychiatry, 55, 1920-1927.

Clark, D. M., Layard, R., Smithies, R., Richards, D. A., Suckling, R., y Wright, B. (2009). Improving access to psychological therapy: Initial evaluation of two UK demonstration sites. Behavior Research and Therapy, 47, 910-920.

Cuijpers, P., van Straten, A., y Andersson, G. (2008). Internet-administered cognitive behavior therapy for health problems: a systematic review. Journal of Behavioral Medicine, 31, 169-177.

De Graaf, L. E., Huibers, M. J. H., Riper, H., Gerhards, S. A. H., y Arntz, A. (2009). Use and acceptability of unsupported online computerized cognitive behavioral therapy for depression and associations with clinical outcome. Journal of Affective Disorders, 116, 227-231.

Ellard, K. K., Fairholme, C. P., Boisseau, C. L., Farchione, T. J., y Barlow, D. H. (2010). Unified protocol for the transdiagnostic treatment of emotional disorders: Protocol development and initial outcome data. Cognitive and Behavioral Practice, 17, 88-101.

Farchione, T. J., Fairholme, C. P., Ellard, K. K., Boisseau, C. L., Thompson-Hollands, J., y cols. (2012). Unified Protocol for Transdiagnostic Treatment of Emotional Disorders: A Randomized Controlled Trial. Behavior Therapy, 43, 666-678.

Hollon, S. D., y Ponniah, K. (2010). A review of empirically supported psychological therapies for mood disorders in adults. Depression and Anxiety, 27, 891-932.

Kaltenthaler, E., Sutcliffe, P., Parry, G., Beverley, C., Rees, A., y Ferriter, M. (2008) The acceptability to patients of computerized cognitive behaviour therapy for depression: a systematic review. Psychological Medicine, 38, 1521-1530.

Kessler, R. C., Berglund, P., Demler, O., Jin, R., Merikangas, K. R., y Walters, E. E. (2005). Lifetime Prevalence and Age-of-onset Distributions of DSM-IV Disorders in the National Comorbidity Survey Replication. Archives of General Psychiatry, 62, 593-602.

Kooistra, L. C., Wiersma, J. E., Ruwaard, J., an Oppen, P., Smit, F.; Lokkerbol, J.; y cols. (2014). Blended vs. face-to-face cognitive behavioural treatment for major depression in specialized mental health care: Study protocol of a randomized controlled cost-effectiveness trial. BMC Psychiatry, 14, 290.

Linehan, M. M. (2003). Manual de tratamiento de los trastornos de personalidad límite. Barcelona: Paidós.

McEvoy, P. M., Nathan, P., y Norton, J. (2009). Efficacy of transdiagnostic treatments: a review of published outcome studies and future research directions. Journal of Cognitive Psychothery, 23, 27-40.

Nathan, P. E., y Gorman, J. M. (2007). A guide to treatments that work. 3rd edition. Nueva York: Oxford University Press.

Norton, P. J, Price, E. P. (2007). A meta-analytic review of cognitive-behavioral treatment outcome across the anxiety disorders. The Journal of Nervous and Mental Disease, 195, 521 531. 
Olatunji, B. O., Cisler, J. M., y Deacon, B. J. (2010). Efficacy of cognitive behavioral therapy for anxiety disorders: a review of meta-analytic findings. Psychiatric Clinics of North America, 33, 557-577.

Osgood-Hynes, D. J., Greist, J. H., Marks, I. M., Baer, L., Heneman, S. W., Wenzel, K. y cols. (1998). Self-administered psychotherapy for depression using a telephone-accessed computer system plus booklets: an open US-UK study. Journal of Clinical Psychiatry, 59, 358-365.

Richards, D., y Richardson, T. (2012). Computer-based psychological treatments for depression: a systematic review and meta-analysis. Clinical Psycholy Review, 32, 329-342.

Sheehan, D. V., Lecrubier, Y., Sheehan, K. H., Amorim, P., Janavs, J., Weiller, E., y cols. (1998). The Mini-International Neuropsychiatric Interview (MINI): the development and validation of a structured diagnostic psychiatric interview for DSM-IV and ICD-10. Journal of Clinical Psychiatry, 59, 34-57.

Wittchen, H. U., Jacobi, F., Rehm, J., Gustavsson, A., Svensson, M., Jönsson, B., y cols. (2010). The size and burden of mental disorders and other disorders of the brain in Europe. European Neuropsychopharmacology, 21, 655-679. 